\title{
ON A RESULT OF HUA FOR CUBIC POLYNOMIALS ${ }^{1}$
}

\author{
ALVIN SUGAR
}

In a paper by L. K. Hua, ${ }^{2}$ we find the following principal result.

Theorem. For any positive integer $\epsilon$, every integer can be expressed in infinitely many ways as a sum of seven values of the cubic function

$$
f(x)=\epsilon\left(x^{3}-x\right) / 6+x
$$

for integral values of $x$; also, every integer can be expressed in infinitely many ways as a sum of seven values of

$$
F(x)=\left(x^{3}-x\right) / 6
$$

for integral values of $x .^{3}$

In this paper we get a better result by applying a known identity for cubes to generalizations of the above polynomials. We state our results in the following two theorems. Note that, in Theorem $1, \epsilon$ may be positive or negative, or, for that matter, zero.

Unless otherwise stated all letters in this paper stand for integers, positive, negative, or zero.

Theorem 1. For any $\epsilon, c$, and any $k$ prime to $\epsilon$, every integer can be expressed in infinitely many ways as a sum of five values of the function

$$
p(x)=\epsilon\left(x^{3}-x\right) / 6+k x+c
$$

for integral values of $x$.

THEOREM 2. For any $k$ and $c$, every integer can be expressed as a sum of four values of the function

$$
P(x)=\left(x^{3}-x\right) / 6+k x+c
$$

for integral values of $x$.

Theorem 1 is trivially true when $\epsilon=0$. For, in this case, $(\epsilon, k)=1$ implies $k=1$. Henceforth we take $\epsilon \neq 0$.

${ }_{1}$ Presented to the Society, April 27, 1940.

${ }^{2}$ On the representation of integers by the sums of seven cubic functions, Tôhoku Mathematical Journal, vol. 41 (1935-1936), pp. 361-366.

${ }^{3}$ Hua fails to mention in his formulation of this theorem whether his $\epsilon$ may take negative values. It seems that Hua implicitly assumed $\epsilon$ positive, as was noted by Pall in his review of the Hua paper in the Zentralblatt für Mathematik, vol. 14, p. 10. (This assumption was probably unnecessary, however.) 
Let us first prove Theorem 1 for Hua's polynomial $f(x)$. Since $x^{3}-x$ is divisible by 6 , it is evident that

$$
\frac{\epsilon}{6}\left(x^{3}-x\right)+x \equiv x(\bmod |\epsilon|),
$$

for any integer $x$. Hence it is evident that there exist $|\epsilon|$ incongruent values modulo $|\epsilon|$ of $f$. Consequently for any integer $n$ it is true that there exist integers $t$ and $m$ such that

$$
n=f(t)+m \epsilon \text {. }
$$

We can show that $m \epsilon$ is a sum of four values of $f$ by employing the following identity:

$$
f(m+1)+f(m-1)+2 f(-m)=m \epsilon .
$$

From (2) and (3), we get

$$
n=f(t)+f(m+1)+f(m-1)+2 f(-m),
$$

which tells us that every integer is a sum of five values of $f(x), x$ integral. Since we can replace $t$ in (2) by any number congruent to it modulo $|\epsilon|$, it follows that the representation (4) can be made in infinitely many ways.

With but slight modification of this proof, we can obtain the more general result stated in Theorem 1 . In this case (1) becomes $p(x) \equiv k x+c(\bmod |\epsilon|)$. And, since $k$ is prime to $\epsilon, k x+c$ ranges over a complete set of residues modulo $|\epsilon|$ when $x$ does. Now if we replace $f$ by $p$ in (3), the right member of this identity becomes $m \epsilon+4 c$. From these facts we can deduce Theorem 1 .

If we replace $f$ by $P$ in (3), the right member of our identity becomes $m+4 c$, which ranges over all of the integers with $m$; hence we have proved Theorem 2 . It is evident from Theorem 1 , with $\epsilon=1$, that we could duplicate Hua's "infinitely many ways" in our Theorem 2 if we increased the number of summands to five.

Cooper Union Institute of Technology 